\title{
Healing of Achilles tendon partial tear following focused shockwave: a case report and literature review
}

This article was published in the following Dove Press journal:

Journal of Pain Research

19 May 2017

Number of times this article has been viewed

\author{
Yu-Chun Hsu',* \\ Wei-Ting $\mathrm{Wu}^{2, *}$ \\ Ke-Vin Chang ${ }^{2-4}$ \\ Der-Sheng $\mathrm{Han}^{2-4}$ \\ Li-Wei Chou ${ }^{5-7}$ \\ 'Department of Physical Medicine \\ and Rehabilitation, Taipei Veterans \\ General Hospital, ${ }^{2}$ Department of \\ Physical Medicine and Rehabilitation \\ and Community and Geriatric \\ Research Center, National Taiwan \\ University Hospital, Bei-Hu Branch, \\ ${ }^{3}$ National Taiwan University College \\ of Medicine, ${ }^{4}$ Community and \\ Geriatric Research Center, National \\ Taiwan University Hospital, Bei-Hu \\ Branch, Taipei, ${ }^{5}$ Department of \\ Physical Medicine and Rehabilitation, \\ China Medical University Hospital, \\ ${ }^{6}$ Graduate Institute of Acupuncture \\ Science, College of Chinese \\ Medicine, China Medical University, \\ ${ }^{7}$ Department of Rehabilitation, Asia \\ University Hospital, Taichung, Taiwan \\ *These authors contributed equally to \\ this work
}

Correspondence: Li-Wei Chou Department of Physical Medicine and Rehabilitation, China Medical University Hospital, No 2 Yuh-Der Road, Taichung 40447, Taiwan

Tel +886 42205212 I (Extension 238I)

Fax +886 42202604 I

Email chouliwe@gmail.com

\begin{abstract}
Achilles tendinopathy is a common cause of posterior heel pain and can progress to partial tendon tear without adequate treatment. Effects of traditional treatments vary, and many recent reports focus on the use of extracorporeal shockwave therapy (ESWT) for Achilles tendinopathy but not for Achilles tendon partial tear. Here, we report the case of a 64-year-old female suffering from severe left heel pain for half a year. All treatment and rehabilitation were less effective until ESWT was applied. Each course of focused shockwave therapy included 2500 shots with energy flux density from $0.142 \mathrm{~mJ} / \mathrm{mm}^{2}$ to $0.341 \mathrm{~mJ} / \mathrm{mm}^{2}$. The visual analog scale decreased from nine to one degree. High-resolution musculoskeletal ultrasonography was performed before and 1 month after the treatment, which revealed healing of the torn region and decrease in inflammation. ESWT had shown to be an alternative treatment for Achilles tendon partial tear under safety procedure and ultrasound observation.
\end{abstract}

Keywords: focused shockwave, Achilles tendon, partial tear, ultrasonography

\section{Introduction}

Achilles tendinopathy, a common cause of posterior heel pain, swelling, and loss of function, is originated from excessive loading-induced injury, degeneration, medicine, or systemic disease. ${ }^{1}$ Running and jumping are the most frequent contributors, which still can be seen in sedentary individuals. ${ }^{2}$ Without adequate treatment, it may progress to partial tendon tear because of degeneration changing, ${ }^{1}$ and the activities of daily living or sporting movement can both be interfered.

Treatments consist of rest, use of systemic nonsteroidal anti-inflammatory drugs, and stretching exercises but the evidence of effectiveness is limited. ${ }^{3}$ Recently, many reports focused on eccentric training ${ }^{4,5}$ and other treatments like thermal and electrical therapies, massage techniques, steroid injections, and instillation of platelet-rich plasma have all been described as potentially useful non-operative interventions. ${ }^{6}$

Extracorporeal shockwave therapy (ESWT) has been widely used for the treatment of calcific lesions and tendinopathy in recent years. Some reports claim that ESWT enables continuation of activity during recovery through short-term analgesic effect and longer term resolution of the pathology. ${ }^{7}$ Due to ESWT has been used for the treatment of Achilles tendinopathy, ${ }^{7}$ with specific improvement in reports by Vulpiani et $\mathrm{al}^{8}$ but no article focused on more severe problems like tear, we try using focused shockwave on a case with left side partial tear of the Achilles tendon. 


\section{Case description}

A 64-year-old female nurse suffered from severe left heel pain for half year after a long period of walking and standing. She mentioned that the dull pain was induced after ankle sprain with a "pop" sound when traveling in a bus, and she still could walk at that time. She did not receive any treatment or take rest at that time. Two days later, the pain became severe and radiated to soleus muscle, especially when walking, accompanied with sharp pain sensation especially when climbing stairs. Swelling around the heel to leg was also noted with mild ecchymosis at that time. Before visiting, she received cold or hot packing, electric therapy, stretching exercising, manual therapy, massage, acupuncture, oral and topical medicines at other clinics. All modes of treatment were less effective and the symptoms progressed.

Half a year later, she could not even walk or stand when first visiting at our department. The activity of daily living (ADL) and working performance were affected. The visual analog scale (VAS) was nine. At the first visit, granulation like lesions and tenderness was observed at surface of the heel skin, compatible to Haglund's deformity and spurs formation as revealed on the image taken through X-ray. No bony fracture was noted at tibia, fibula, or calcaneus bone. Active ankle dorsiflexion and plantar flexion would induce painful sensation during examination. Passive range of motion could be smoothly completed when gently performed on the ankle, but positive finding of Thompson test was noted when the calf was squeezed. Pain would also generate when the area around the insertion site of Achilles tendon was compressed and knocked. Taut band with referred pain to the ankle and twitch sign was noted at soleus muscle. Soft tissues around the heel showed mild swelling, but no wound or ecchymosis was noted at that time. Muscle power of the lower limb was within normal limit except at active plantar flexion compared to the healthy side. Because the muscle power was of grade 3 , lesions at common tendon of soleus and gastrocnemius should be suspected. According to the aforementioned findings, Achilles tendinitis, retrocalcaneal bursitis, tendon tear, soleus and gastrocnemius muscle strain, and myofascial pain syndrome should be distinguished.

Musculoskeletal ultrasonography was performed with a Esaote MyLab ${ }^{\mathrm{TM}}$ Gamma machine (Esaote S.p.a., Florence, Italy) and a high-resolution linear array transducer with 12 $\mathrm{MHz}$ frequency. The following examinations were performed with the same machine and the same physician except fourth time due to machine maintenance. The images indicated retrocalcaneal bursitis, multiple spurs, and calcified lesions around the calcaneus. The Achilles tendon was swollen from "watershed" region, with some hypoechoic lesions between it and insertional site. The thickness of the tendon was $12.9 \mathrm{~mm}$, and the locations of multiple partial tears were $1 \mathrm{~cm}$ proximal to the calcaneus (Figure 1A). Tears were scattered along the tendon base. Blood flow was increased along the tendon as revealed under power Doppler view (Figure 1B). No hematoma, scars, and laceration were noted at soleus and gastrocnemius. No tendinitis or tear was noted at peroneal tendon, posterior tibialis, flexor digitorum longus, and flexor hallucis longus. All ligaments around the ankle were checked at the same time, and no sprain or tear was detected.

As the patient was diagnosed with Achilles tendon partial tear combined with calcified tendinitis, Haglund's deformity, and myofascial pain syndrome at soleus, she received six sessions of low-power laser therapy, ultrasound, and transcutaneous electrical nerve stimulation at first, but she did get any pain relief. Survey of musculoskeletal ultrasound at the second visit was carefully performed in initial with the same machine and $18 \mathrm{MHz}$ linear array transducer to build up the baseline image (Figure 2A and B). In this survey, more hypervascularity pattern was noted than in the previous examination.

Then, she received focused shockwave therapy for eight times with a Richard Wolf and ELvation-Piezo Wave 2 machine (ELvation Medical GmbH, Kieselbronn, Germany).

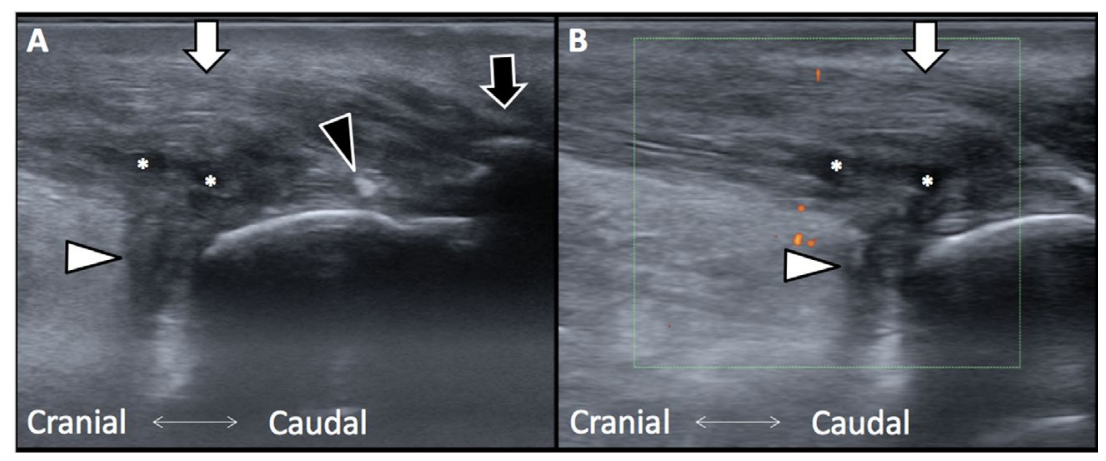

Figure I (A) Achilles tendon swelling with partial tear. (B) Increased blood flow as revealed with orange dots under power Doppler views. Asterisks, tear site; black arrowhead, calcified lesions; white arrowhead, retrocalcaneal bursa; black arrow, spurs; white arrow, Achilles tendon. 


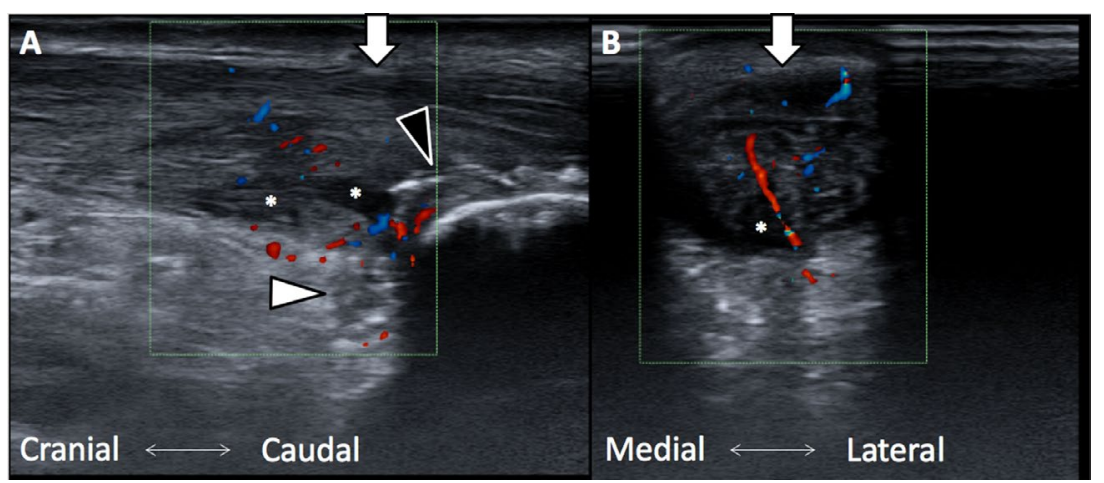

Figure 2 (A) Achilles tendon swelling, partial tear, and retrocalcaneal bursitis. Increased blood flow as revealed under color Doppler view. (B) Achilles tendon swelling and partial tear in a transverse view. Asterisks, tear site; black arrowhead, calcified lesions; white arrowhead, retrocalcaneal bursa; white arrow, Achilles tendon. Increased blood flow is shown with red and blue dots as revealed under color Doppler view.

Focused shockwave therapy for the first three times was carried out as per the sessions summarized by Schmitz et $\mathrm{al}^{9}$ and the last five courses were performed for patient satisfaction. Each course of treatment included 2500 shots performed by the same physician without any local anesthesia introduced previously. The total period of the treatment was 3 months with the first three courses arranged every 2 weeks under consideration of the compliance to the soreness of treatment. The other five courses were performed once a week. First 500 shots with a $30 \mathrm{~mm}$ gel pad were performed on the soleus muscle to relieve the myofascial pain syndrome and muscle guarding; another 2000 shots with a $10 \mathrm{~mm}$ gel pad were performed on the calcified lesions, tear sites, and insertion site of Achilles tendon. The initial intensity level of the machine was five and 12 at terminal at both areas, conversing energy flux density (EFD) from $0.142 \mathrm{~mJ} / \mathrm{mm}^{2}$ to $0.341 \mathrm{~mJ} / \mathrm{mm}^{2}$ and peak pressure from 16.9 MPa to $36.0 \mathrm{MPa}$. The frequency of shots was regularized at $6 \mathrm{~Hz}$. Rapid but careful survey with ultrasound was conducted before and after each course to prevent any risk of tear due to the procedure.

During the treatment, no friction massage or strengthening or stretching exercise was prescribed. She still received local treatment with low-power laser therapy on Achilles tendon insertion site and transcutaneous electrical nerve stimulation on soleus as usual without administration of any injection, medicine, and topical medicine or immobilization through orthosis or bandage. Any abrupt movement, jumping, or running was prohibited. The pain was much relieved just after the first course of treatment, with a decrease in VAS from nine to four. The pain sensation would rebound to a score of six on the VAS several days later after the first three courses, but was maintained at a score of two to three on the VAS during four to six courses of treatment. Two months after the first course, we performed musculoskeletal ultrasonography again. The Achilles tendon swelling and partial tear were still noted, accompanied with much increased blood flow along the tendon as revealed under color Doppler view (Figure 3A and B).

After eight times of treatment, she mentioned that the pain was near fully relieved and the VAS decreased to one. ADL also improved from dependent to independent, ${ }^{10}$ as noted on the transfer scale and she could return to work, climb the floors, and even go to the supermarket like before. No painful sensation would be induced when standing, bending, or performing a range of motion, with only mild tenderness at Achilles tendon insertion site. The physical examination performed by the same method and physician showed negative finding on squeeze test of soleus and active or passive range of motion of the ankle.

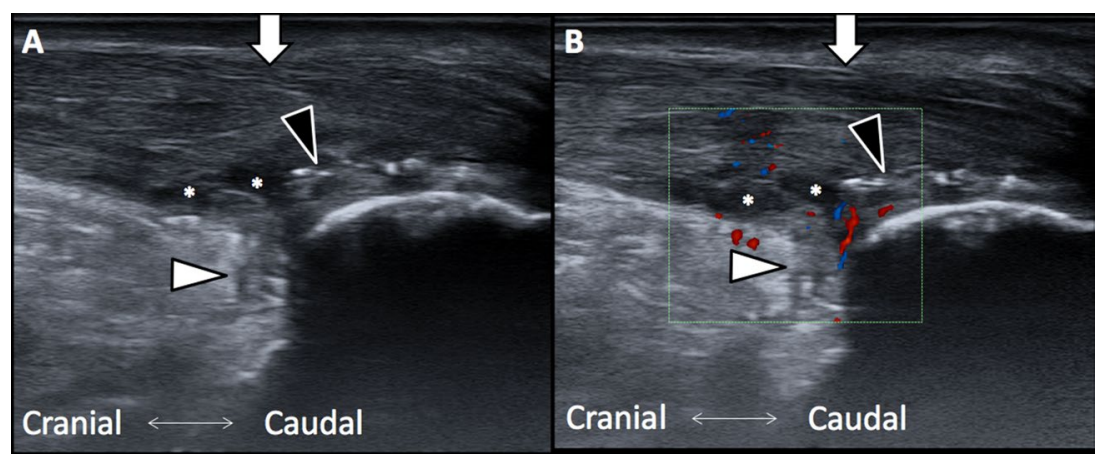

Figure 3 (A) Achilles tendon swelling, partial tear, and retrocalcaneal bursitis. (B) Increased blood flow in longitudinal view. Asterisks, tear site; black arrowhead, calcified lesions; white arrowhead, retrocalcaneal bursa; white arrow, Achilles tendon. 


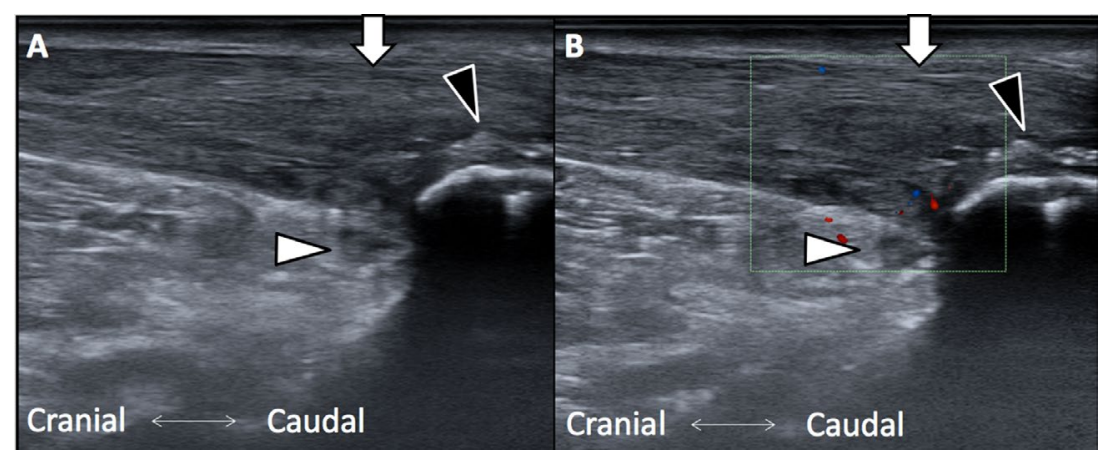

Figure 4 (A) Achilles tendon swelling and retrocalcaneal bursitis. Healing pattern was noted at tear site. (B) Inflammation was much relieved as revealed under color Doppler view. Black arrowhead, calcified lesions; white arrowhead: retrocalcaneal bursa; white arrow, Achilles tendon.

The fourth musculoskeletal ultrasonography with a different machine (GE logic E and linear transducer of $12 \mathrm{MHz}$; General Electric Company, Boston, MA, USA) was performed 3 months after the first course of treatment. The tendon swelling was mildly relieved, and the hypoechoic lesions were much improved. In order to rule out the bias due to the use of a different machine, we performed fifth musculoskeletal ultrasonography with the same machine and transducer as the first three times 1 month later after the last treatment. The same focus, depth, frequency, and bony landmark used in the second examination were chosen. The fiber filament was noted in the previous hypoechoic lesions (Figure 4A and $\mathrm{B}$ ), and the blood flow around the tendon was much relieved. Although multiple calcified lesions, spurs, and tendinopathy were still noted, healing of Achilles tendon partial tear was confirmed. Previous positive signs of Achilles tendinopathy, such as, pain on dorsiflexion and plantar flexion, or positive findings of Thompson test, could not be detected again with normal active and passive range of motion under examination. No painful sensation was mentioned when walking and climbing except mild tightness. The effect of focused shockwave therapy was still continued for more than 4 months after the final course of focused shockwave therapy. The patient provided written informed consent to use the case details and any accompanying images published.

\section{Discussion}

In our report, the Achilles tendon partial tear was under healing process and the of quality of life also improved after several times of focused shockwave therapy. The findings inspire the possibility of another choice of treatment for Achilles tendinopathy or partial tear if we fully understand the mechanism.

Degeneration and synthesis imbalance of the matrix when aging, ${ }^{11}$ overusing with repetitive wear and tear, or weight bearing movements can induce Achilles tendon pain during daily activities. Achilles tendinopathy can localize at paratendon region, insertional site, or $2-6 \mathrm{~cm}$ proximal to the insertion site as the "watershed" region. The symptoms may become severe without adequate rest and treatment. Systemic nonsteroidal anti-inflammatory drugs, ${ }^{12}$ transcutaneous electrical nerve stimulation, eccentric training, ${ }^{4,5}$ heavy slow resistance training, ${ }^{13}$ laser, ultrasound, ${ }^{12}$ and massage have been described as potentially useful methods. As mentioned for interventional choice, radiofrequency therapies, plateletrich plasma injections, local glucocorticosteroid injections, or surgery ${ }^{12}$ are chosen for chronic cases.

The etiology of Achilles tendon tear including sports, ${ }^{14}$ pre-existing tendinopathy, ${ }^{15}$ degenerative changes,${ }^{16}$ fluoroquinolone use, ${ }^{17}$ corticosteroid use, ${ }^{18}$ and unknown reason should be taken into consideration. When tear is noted, operation or cast immobilization shall be considered.

Recently, ESWT has been found as an alternative option if conservative treatment fails prior to surgical interventions. Most reports show evidence of therapeutic benefits of ESWT in calcific rotator cuff tendinopathy ${ }^{19}$ and plantar fasciitis, ${ }^{20}$ and it has been found to be more effective than traditional methods on chronic patellar tendinopathy. ${ }^{21}$ In recommended energy densities, minor complications of ESWT like pain and local swelling without structural damage have been reported $;^{22,23}$ skin erosions, hematoma, or nerve lesions have also been noted. ${ }^{19}$

On the other hand, some reviews report that controlled randomized trials of ESWT did not result in significant improvement. ${ }^{24}$ For treatment of Achilles tendinopathy, ESWT also can be considered, but we shall pay more attention to the complications that may be induced because of the weight bearing of the whole body. Some researchers claim that tendinopathy improves by at least 1 year after treatment without adverse effects, ${ }^{25}$ but they exclude the case of tendon tear at first. In a report by Lin et al, risk of Achilles tendon tear due to ESWT was suspected. ${ }^{26}$ The case once received calcaneal osteotomy 2 years ago and an unknown course of triamcinolone injection 1 year ago. ${ }^{26}$ Tendon fragility and weakness of surrounding tissues should be taken into con- 
sideration. Another important article showed the use of shock wave on 49 patients with chronic Achilles tendon pain, but two of them in treatment group suffered from tendon rupture. ${ }^{27}$ The two cases only received one time or treatment, and both happened after jumping or stepping movement which should be forbidden even in the control group. Besides, the article did not mention about how to distinguish Achilles tendon partial tear from tendinopathy, and no ultrasound examination or magnetic resonance imaging was performed but the cases were diagnosed only through physical examination. Following up research should be considered as technology progresses. In our case, healing is noted otherwise; so, completely mechanical survey shall be systemic reviewed.

Traditionally, the mechanical effect is defined as increasing pressure for fragmentation of the lesion, inflammatory response from ESWT or deposit fragmentation, and analgesic effect on nociceptors through blocking the gate control mechanism. ${ }^{19}$ Increased vascularization and ultimately phagocytosis of the deposit can help in the healing of the tissues. ${ }^{19}$ Alteration of cell activity through cavitation, acoustic microstreaming, and cell membrane permeability should also be reported. ${ }^{28}$ Other report focuses on the substance P level at treated site, which increased initially and then decreased, explaining the initial pain during treatment and subsequent lasting pain relief. ${ }^{29}$

Focused shockwave concentrates three-dimensional pressure pulses into small focal areas in order to optimize therapeutic effects and minimize effects on surrounding tissues. ${ }^{28}$ In clinical, EFD applies ranges from 0.001 to $0.4 \mathrm{~mJ} / \mathrm{mm}, 2,30$ and at lower and medium EFD, nitric oxide is released with antalgic, angiogenetic and anti-inflammatory effects, which will help tissues healing and pain relieving. ${ }^{30}$ In our case, the EFD was increased from $0.142 \mathrm{~mJ} / \mathrm{mm}^{2}$ to $0.341 \mathrm{~mJ} / \mathrm{mm}^{2}$, and the treatment effect may partially be explained from the aforementioned theory.

Some researchers report that ESWT enhances cell proliferation and migration of cultured tenocytes from human, ${ }^{31}$ which represents only $5 \%$ of normal tissue volume but with function for their maintenance and repair. ${ }^{32}$ When under mechanical stimulation like ESWT, tenocytes can increase $T G F-b 1$ gene expression. ${ }^{33}$ During wound healing, $T G F-b 1$ can inhibit inflammation and extracellular matrix degradation because of macrophages ${ }^{34}$ For tissue-derived stem cells, recruitment, proliferation, and differentiation may also be enhanced through ESWT treatment. ${ }^{35,36}$

In our case report, according to the ultrasonography performed by the same physician, healing process of partial tear can be identified. The quality of life can also become much better than before and the effect can continue for more than 4 months after the final course of therapy. We believe that these theories can be confirmed with our report in clinic.

\section{Conclusion}

Our report shows a case with Achilles tendon partial tear and poor response to previous treatment. After the application of ESWT under safety conditions and observation of the results of musculoskeletal ultrasound, the pain was found to be much relieved and healing of the torn region was revealed. After further research, the treatment may become another choice for Achilles tendon partial tear.

\section{Acknowledgments}

This work was supported in part by Taiwan Ministry of Health and Welfare Clinical Trial Center (MOHW106-TDUB-212-113004), CMU under the Aim for Top University Plan of the Ministry of Education, Taiwan, and National Taiwan University Hospital, Bei-Hu branch (Beihu 10603).

\section{Disclosure}

The authors report no conflicts of interest in this work.

\section{References}

1. Järvinen TA, Kannus P, Maffulli N, Khan KM. Achilles tendon disorders: etiology and epidemiology. Foot Ankle Clin. 2005;10(2):255-266.

2. Rompe JD, Nafe B, Furia JP, Maffulli N. Eccentric loading, shock-wave treatment, or a wait-and-see policy for tendinopathy of the main body of tendo Achilles: a randomized controlled trial. Am J Sports Med. 2007; 35(3):374-383.

3. Magra M, Maffulli N. Nonsteroidal antiinflammatory drugs in tendinopathy: friend or foe. Clin J Sport Med. 2006;16(1):1-3.

4. Mansur NS, Faloppa F, Belloti JC, et al. Shock wave therapy associated with eccentric strengthening versus isolated eccentric strengthening for Achilles insertional tendinopathy treatment: a double-blinded randomised clinical trial protocol. BMJ Open. 2017;7(1):e013332.

5. Balius R, Álvarez G, Baró F, et al. A 3-arm randomized trial for Achilles tendinopathy: eccentric training, eccentric training plus a dietary supplement containing mucopolysaccharides, or passive stretching plus a dietary supplement containing mucopolysaccharides. Curr Ther Res Clin Exp. 2016;78:1-7.

6. Sayana MK, Maffulli N. Insertional Achilles tendinopathy. Foot Ankle Clin. 2005;10:309-320.

7. Furia JP. High-energy extracorporeal shock wave therapy as a treatment for chronic noninsertional Achilles tendinopathy. Am J Sports Med. 2008;36(3):502-508.

8. Vulpiani MC, Trischitta D, Trovato P, Vetrano M, Ferretti A. Extracorporeal shockwave therapy (ESWT) in Achilles tendinopathy. A longterm follow-up observational study. J Sports Med Phys Fitness. 2009; 49(2):171-176.

9. Schmitz C, Császár NB, Milz S, et al. Efficacy and safety of extracorporeal shock wave therapy for orthopedic conditions: a systematic review on studies listed in the PEDro database. Br Med Bull. 2015;116: $115-138$. 
10. Katz S, Ford AB, Moskowitz RW, Jackson BA, Jaffe MW. Studies of illness in the aged. The index of ADL: a standardized measure of biological and psychosocial function. JAMA. 1963;185:914-919.

11. Knobloch K. The role of tendon microcirculation in Achilles and patellar tendinopathy. J Orthop Surg Res. 2008;3:18.

12. Kearney R, Costa ML. Insertional Achilles tendinopathy management: a systematic review. Foot Ankle Int. 2010;31(8):689-694.

13. Beyer R, Kongsgaard M, Hougs Kjær B, Øhlenschlæger T, Kjær M, Magnusson SP. Heavy slow resistance versus eccentric training as treatment for Achilles tendinopathy: a randomized controlled trial. Am J Sports Med. 2015;43(7):1704-1711.

14. Plecko M, Pass1 R. Ruptures of the Achilles tendon: causes and treatment. J Finn Orthop Traumatol. 1991;14:201-204.

15. Riley G. Tendinopathy - from basic science to treatment. Nat Clin Pract Rheumatol. 2008;4(2):82-89.

16. Kvist M, Józsa L, Järvinen M. Vascular changes in the ruptured Achilles tendon and paratendon. Int Orthop. 1992;16(4):377-382.

17. Khaliq Y, Zhanel GG. Fluoroquinolone-associated tendinopathy: a critical review of the literature. Clin Infect Dis. 2003;36(11):1404-1410.

18. Speed CA. Corticosteroid injections in tendon lesions. BMJ. 2001;323:382.

19. Mouzopoulos G, Stamatakos M, Mouzopoulos D, Tzurbakis M. Extracorporeal shock wave treatment for shoulder calcific tendonitis: a systematic review. Skeletal Radiol. 2007;36(9):803-811.

20. Lee SJ, Kang JH, Kim JY, Kim JH, Yoon SR, Jung KI. Dose-related effect of extracorporeal shock wave therapy for plantar fasciitis. Ann Rehabil Med. 2013;37(3):379-388.

21. Wang CJ, Ko JY, Chan YS, Weng LH, Hsu SL. Extracorporeal shockwave for chronic patellar tendinopathy. Am J Sports Med. 2007; 35(6):972-978.

22. Fridman R, Cain JD, Weil L Jr, Weil L Sr. Extracorporeal shockwave therapy for the treatment of Achilles tendinopathies: a prospective study. J Am Podiatr Med Assoc. 2008;98(6):466-468.

23. van Leeuwen MT, Zwerver J, van den Akker-Scheek I. Extracorporeal shockwave therapy for patellar tendinopathy: a review of the literature. Br J Sports Med. 2009;43(3):163-168.

24. Trebinjac S, Mujić-Skikić E, Ninković M, Karaiković E. Extracorporeal shock wave therapy in orthopaedic diseases. Bosn J Basic Med Sci. 2005; $5(2): 27-32$.
25. Saxena A, Ramdath S Jr, O'Halloran P, Gerdesmeyer L, Gollwitzer H. Extra-corporeal pulsed-activated therapy ("EPAT" sound wave) for Achilles tendinopathy: a prospective study. J Foot Ankle Surg. 2011;50(3): 315-319.

26. Lin TC, Lin CY, Chou CL, Chiu CM. Achilles tendon tear following shock wave therapy for calcific tendinopathy of the Achilles tendon: a case report. Phys Ther Sport. 2012;13(3):189-192.

27. Costa ML, Shepstone L, Donell ST, Thomas TL. Shock wave therapy for chronic Achilles tendon pain: a randomized placebo-controlled trial. Clin Orthop Relat Res. 2005;440:199-204.

28. Auge BK, Preminger GM. Update on shock wave lithotripsy technology. Curr Opin Urol. 2002;12(4):287-290.

29. Maier M, Averbeck B, Milz S, Refior HJ, Schmitz C. Substance P and prostaglandin E2 release after shock wave application to the rabbit femur. Clin Orthop Relat Res. 2003;(406):237-245.

30. Loew M, Rompe JD. Stoßwellenbehandlung bei orthopädischen Erkrankungen: mit 8 Tabellen. Stuttgart: Enke; 1997.

31. Vetrano M, d'Alessandro F, Torrisi MR, Ferretti A, Vulpiani MC, Visco V. Extracorporeal shock wave therapy promotes cell proliferation and collagen synthesis of primary cultured human tenocytes. Knee Surg Sports Traumatol Arthrosc. 2011;19(12):2159-2168.

32. Lui PP, Chan KM. Tendon-derived stem cells (TDSCs): from basic science to potential roles in tendon pathology and tissue engineering applications. Stem Cell Rev. 2011;7(4):883-897.

33. Banes AJ, Horesovsky G, Larson C, et al. Mechanical load stimulates expression of novel genes in vivo and in vitro in avian flexor tendon cells. Osteoarthritis Cartilage. 1999;7(1):141-153.

34. Feinberg MW, Jain MK, Werner F, et al. Transforming growth factorbeta 1 inhibits cytokine-mediated induction of human metalloelastase in macrophages. $J$ Biol Chem. 2000;275(33):25766-25773.

35. Catalano MG, Marano F, Rinella L, et al. Extracorporeal shockwaves (ESWs) enhance the osteogenic medium-induced differentiation of adipose-derived stem cells into osteoblast-like cells. JTissue Eng Regen Med. 2017;11(2):390-399.

36. Raabe O, Shell K, Goessl A, et al. Effect of extracorporeal shock wave on proliferation and differentiation of equine adipose tissuederived mesenchymal stem cells in vitro. Am J Stem Cells. 2013;2(1): 62-73.
Journal of Pain Research

\section{Publish your work in this journal}

The Journal of Pain Research is an international, peer reviewed, open access, online journal that welcomes laboratory and clinical findings in the fields of pain research and the prevention and management of pain. Original research, reviews, symposium reports, hypothesis formation and commentaries are all considered for publication.

\section{Dovepress}

The manuscript management system is completely online and includes a very quick and fair peer-review system, which is all easy to use. Visit http://www.dovepress.com/testimonials.php to read real quotes from published authors. 